\title{
Image Resolution Enhancement in Satellite Based On Discrete Wavelet Transform
}

\author{
Bejugam jyothi, G.ramesh
}

\begin{abstract}
Satellite images are being used in many fields of research. One of the major issues of these types of images is their resolution. In this project, I propose a new satellite image resolution enhancement technique based on the interpolation of the high-frequency sub bands obtained by discrete wavelet transform (DWT) and the input image. Resolution of an image has been always an important issue in many image- and videoprocessing applications, such as video resolution enhancement, feature extraction, and satellite image resolution enhancement. Interpolation in image processing is a method to increase the number of pixels in a digital image. Interpolation has been widely used in many image processing applications, such as facial reconstruction,. The DWT operation results in four decomposed sub band images referred to low-low (LL), low-high (LH), high-low (HL), and high-high (HH). The frequency components of those sub bands cover the full frequency spectrum of the original image. The proposed resolution enhancement technique uses DWT to decompose the input image into different sub bands. Then, the high-frequency sub band images and the input low-resolution image have been interpolated, followed by combining all these images to generate a new resolution enhanced image by using inverse DWT. In order to achieve a sharper image, an intermediate stage for estimating the high frequency sub bands has been proposed.
\end{abstract}

Index Terms: Discrete wavelet transform (DWT), interpola- tion, satellite image resolution enhancement, wavelet zero padding (WZP).

\section{Introduction:}

Images are a description of how a parameter varies over a surface. Images may be two-dimensional, such as a photograph, screen display, and as well as a three-dimensional, such as a statue or hologram. They may be captured by optical devices such as cameras, mirrors, lenses, telescopes, microscopes, etc. and natural objects and phenomena, such as the human eye or water surfaces. A digital image is a representation of a twodimensional image using ones and zeros.

Figure 1.1 illustrates the structure of a digital image. The image shown is represented by 40,000 samples arranged in a two-dimensional array of 200 columns by 200 rows. Just as with one- dimensional signals, these rows and columns can be numbered 0 through 199, or 1 through 200. In imaging jargon,

each sample is called a picture element, or in short pixel. Each pixel in this example is a single number between 0 and

255.This value give the brightness of the pixel. Images have their information encoded in the spatial domain, the image equivalent of the time domain. In other words, features in images are represented by edges, not sinusoids

A "typical" digital image is composed of about 500 rows by 500 columns. This is the image quality encountered in television, personal computer applications, and general scientific research. Images with fewer pixels, say 250 by 250 , are regarded as having unusually poor resolution. These low resolution images look noticeably unnatural, and the individual pixels can often be seen. On the other hand, images with more than 1000 by 1000 pixels are considered exceptionally good.

It is common for 256 gray levels (quantization levels) to be used in image processing, corresponding to a single byte per pixel. There are several reasons for this. First, a single byte is convenient for data management, since this is how computers usually store data. Second, the large number of pixels in an image compensate to a certain degree for a limited number of quantization steps.

The value of each pixel in the digital image represents a small region in the continuous image being digitized. For example, imagine that the Venus probe take samples every 10 meters along the planet's surface as it orbits overhead. This defines a square sample spacing and sampling grid, with each pixel representing a 10 meter by 10 meter area. Now, imagine what happens in a single microwave reflection measurement. The space probe emits a highly focused burst of microwave energy, striking the surface in, for example, a circular area 15 meters in diameter. Each pixel therefore contains information about this circular area, regardless of the size of the sampling grid. This region of the continuous image that contributes to the pixel value is called the sampling aperture. The size of the sampling aperture is often related to the inherent capabilities of the particular imaging system being used. 
Color is added to digital images by using three numbers for each pixel, representing the intensity of the three primary colors: red, green and blue. Mixing these three colors generates all possible colors that the human eye can perceive. A single byte is frequently used to store each of the color intensities, allowing the image to capture a total of $256 \times 256 \times 256=16.8$ million different colors.

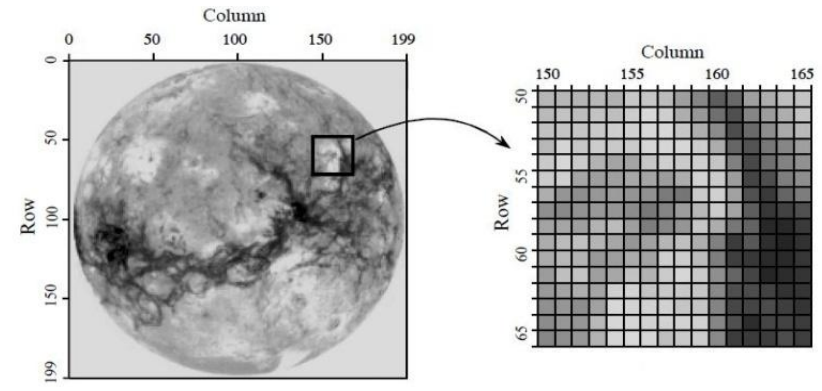

Fig 1.1 planet Venus. Fig1.2 grey levels of a pixel

\subsection{Image processing fundamentals:}

Digital image processing refers processing of the image in digital form. Modern cameras may directly take the image in digital form but generally images are originated in optical form. They are captured by video cameras and digitalized. The digitalization process includes sampling, quantization. Then these images are processed by the five fundamental processes, at least any one of them, not necessarily all of them This section gives various image processing techniques.

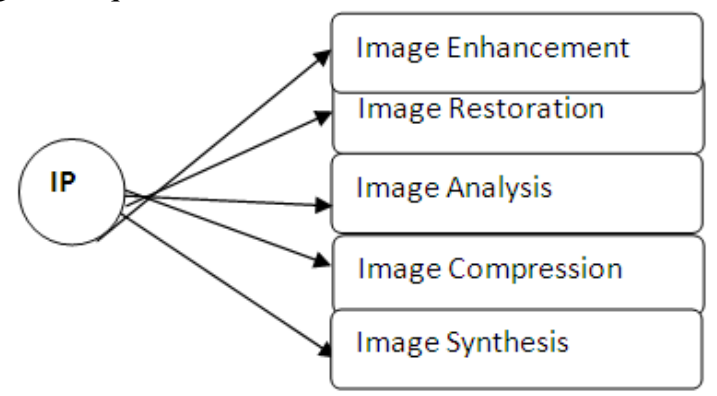

Fig 1.3 Image processing Techniques

\section{Color Models \& Color Image processing:}

The purpose of a color model (also called color space or color system) is to facilitate the specification of colors in some standard, generally accepted way. In essence, a color model is a specification of a coordinate system and a subspace within that system where each color is represented by a single point. The hardware oriented models most commonly used in practice are the RGB model for color monitors and a broad class of color video cameras: the CMY and CMYK models for color printing and the HSI model, which corresponds closely with way humans describe and interpret color. The HSI model also has the advantage that it decouples the color and gray-scale information in an image, making it suitable for many of the gray-scale techniques developed.

The purpose of a color model (also called color space or color system) is to facilitate the specification of colors in some standard, generally accepted way. In essence, a color model is a specification of a coordinate system and a subspace within that system where each color is represented by a single point. The hardware oriented models most commonly used in practice are the RGB model for color monitors and a broad class of color video cameras: the CMY and CMYK models for color printing and the HSI model, which corresponds closely with way humans describe and interpret color. The HSI model also has the advantage that it decouples the color and gray-scale information in an image, making it suitable for many of the gray-scale techniques developed.

\section{The RGB Model}

In the RGB model, each color appears in its primary spectral components of red, green, and blue. This model is based on a Cartesian coordinate system. Images represented in the RGB color model consist of three component images, one for each primary color. When fed into an RGB monitor, these three images combine on the screen to produce a composite color image. The number of bits used to represent each pixel in RGB space is called the pixel depth. Consider an RGB image in which each of the red, green, and blue images is an 8-bit image. Under these conditions each RGB color pixel is said to have a depth of 24 bits. The term full-color image 
is used often to denote a 24-bit RGB color image. The total number of colors in a 24-bit RGB image $16,777,216$.

While high end display cards and monitors provide a reasonable rendition of the colors in a 24-bit RGB image, many systems in use today are limited to 256 colors. Also, there are numerous applications in which it simply makes no sense to use more than a few hundred, and sometimes fewer, colors. A good example of this is provided by the pseudo color image processing techniques. Given the variety of systems in current use, it is of considerable interest to have a subset of colors that are likely to be reproduced faithfully, reasonably independently of viewer hardware capabilities. This subset of colors is called the set of safe RGB colors, or the set of all system safe colors. In Internet applications, they are called safe Web colors or safe browser colors. On the assumption that 256 colors is the minimum number of colors that can be reproduced faithfully by any system in which a desired result is likely to be displayed, it is useful to have an accepted standard notation to refer to these colors.

In the RGB model, each color appears in its primary spectral components of red, green, and blue. THSI model is based on a Cartesian coordinate system. The color subspace of interest is the cube shown in which RGB values are at three corners; cyan, magenta ,and yellow are at three other corners ;black is at the origin; and white is at the corner farthest from the origin. In HSI model, the gray scale (points of equal RGB values) extends from black to white along the line joining these two points. The different colors in HSI model are points on or inside the cube, and are defined by vectors extending from the origin.

Figure: Schematic of the RGB color cube, Points along the main diagonal have gray values, from black at the origin to white at point $(1,1,1)$.

For Convenience, the assumption is that all color values have been normalized so that the cube shown in Fig 3.1 is the unit cube. That is, all values of $R, G$ and $B$ are assumed to be in range $[0,1]$. Image represented in the RGB color model consist of three component images, one for each primary color. When fed into an RGB monitor, these three images combine on the phosphor screen to produce a composite color image. The number of bits used to represent each pixel in RGB space is called pixel depth. Consider an RGB image in which each of the red, green and a blue image is an 8- bit image. Under these conditions each RGB color pixel [that is , a triplet of values (R,G,B)] is said to have a depth of 24 bits ( 3 image planes times the number of bits per plane) . The total number of color in a 24-bit RGB image is (28)3=16.777.215. Figure 3.2 shows the 24-bit RGB color cube corresponding to the diagram I Fig.3.1. It is of interesting to note that acquiring a color images is basically the process shown in Fig.2.3 in reverse. A color image can be acquired by using three filters, sensitive to red, green and blue, respectively. When we view a color scene with a monochrome camera equipped with one of these filters, the result I s a monochrome image whose intensity is proportional to the response of that filter.

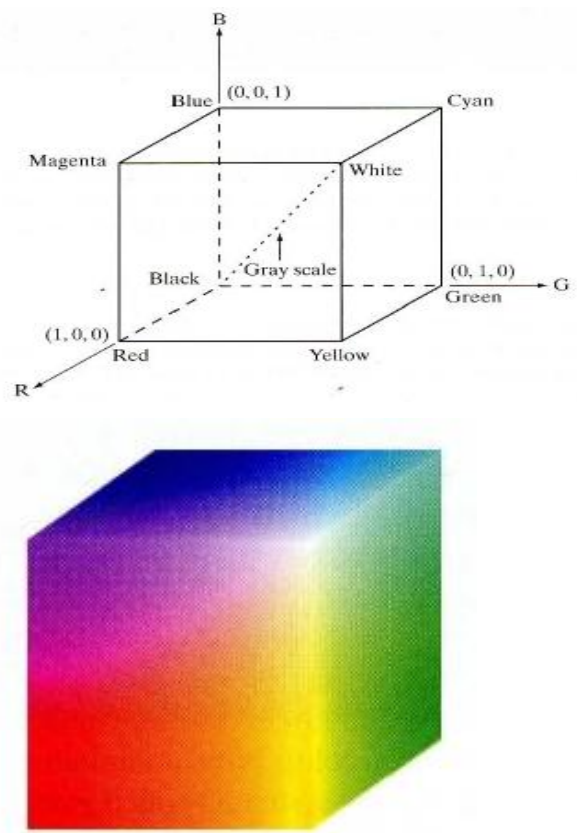

Figure: RGB 24-bit color cube. 


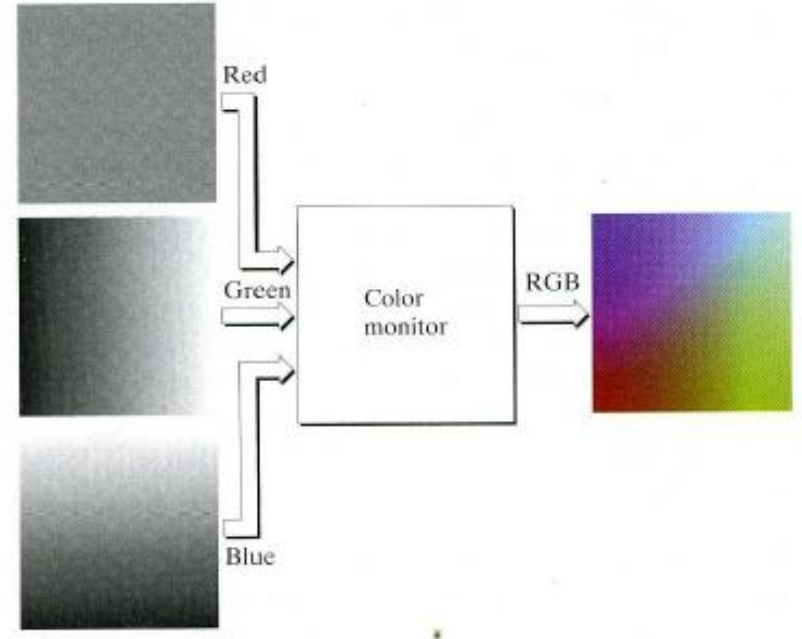

(a)

Figure: (a) Generating the RGB image of the cross- sectional color plane $(127, \mathrm{~GB})$.

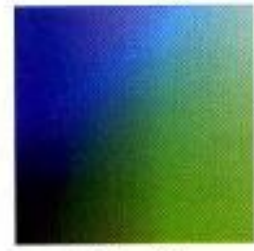

$(R=0)$

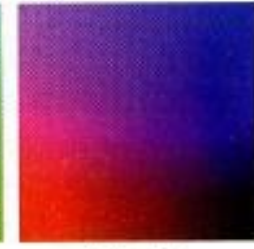

$(G=0)$

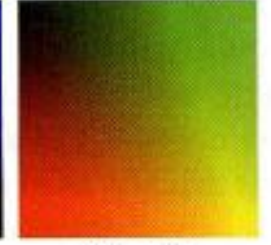

$(B=0)$ (b)

Figure-: (b) The three hidden surface planes in the color cube of .

Repeating HSI process with each filter produces three monochrome images that are the RGB component images of the color scene.( In practice, RGB color image sensors usually integrate HSI process into a single device).Clearly, displaying these three RGB component images in the form shown in Fig.1.4 (a) would yield an RGB color rendition of the original color scene.

\section{Proposed Algorithm:}

In this project, we propose a resolution enhancement technique using interpolated DWT high frequency sub band images and the input low resolution image. Inverse DWT (IDWT) has been applied to combine all these images to generate the final resolution enhanced image. In order to achieve a sharper image, we propose to use an intermediate stage for estimating the high frequency sub bands by utilizing the difference image obtained by subtracting the input image and its interpolated LL sub band. It is necessary to recall that in this project the resolution enhancement is used as a process that enlarges the given input in the way that the output is sharper.In all steps of the proposed satellite image resolution enhancement technique, Daubechies (db.9/7) wavelet transform a smother wavelet function and bicubic interpolation as interpolation technique have been used.

As it was mentioned before, resolution is an important feature in satellite imaging, which makes the resolution enhancement of such images to be of vital importance as increasing the resolution of these images will directly affect the performance of the system using these images as input. The main loss of an image after being resolution enhanced by applying interpolation is on its high-frequency components, which is due to the smoothing caused by interpolation. Hence, in order to increase the quality of the enhanced image, preserving the edges is essential. In this paper, DWT [19] has been employed in order to preserve the high-frequency components of the image. DWT separates the image into different sub band images, namely, LL, LH, HL, and $\mathrm{HH}$. A high-frequency subband contains the high- frequency component of the image. The interpolation can be applied to these four sub band images. In the wavelet domain, the low-resolution image is obtained by low-pass filtering of the high-resolution image .The low- resolution image (LL sub band), without quantization (i.e., with double-precision pixel values) is used as the input for the proposed resolution enhancement process. In other words, low- frequency sub band images are the low resolution of the original image. Therefore, instead of using low-frequency sub band images, which contains less information than the original input image, we are using this input image through the interpolation process. Hence, the input low-resolution image is interpolated with the half of the interpolation factor, $\boldsymbol{\alpha} / 2$, used to interpolate the high-frequency sub bands, as shown in 


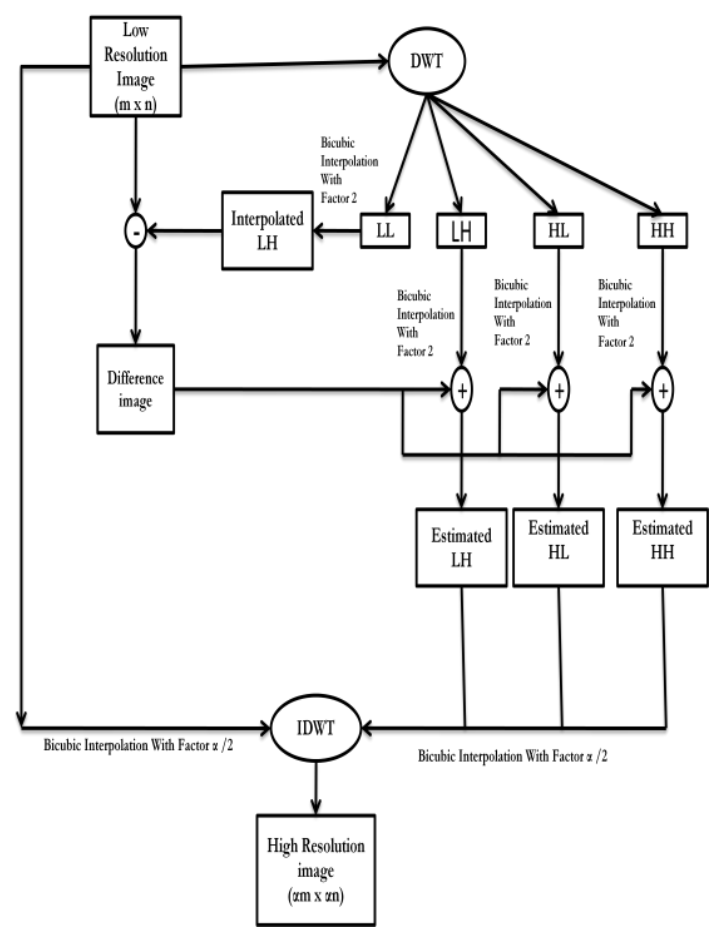

Fig: Block diagram of the proposed resolution enhancement algorithm.

In order to preserve more edge information, i.e., obtaining a sharper enhanced image, we have proposed an intermediate stage in high- frequency sub band interpolation process. As shown in, the lowresolution input satellite image and the interpolated LL image with factor 2 are highly correlated. The difference between the LL sub band image and the low-resolution input image are in their high-frequency components. Hence, this difference image can be use in the intermediate process to correct the estimated highfrequency components. This estimation is performed by interpolating the high-frequency sub bands by factor 2 and then including the difference image (which is high-frequency components on low-resolution input image) into the estimated high-frequency images, followed by another interpolation with factor $\alpha / 2$ in order to reach the required size for IDWT process. The intermediate process of adding the difference image, containing high-frequency components, generates significantly sharper and clearer final image.

This sharpness is boosted by the fact that, the interpolation of isolated high-frequency components in $\mathrm{HH}, \mathrm{HL}$, and $\mathrm{LH}$ will preserve more high-frequency components than interpolating the low-resolution image directly.

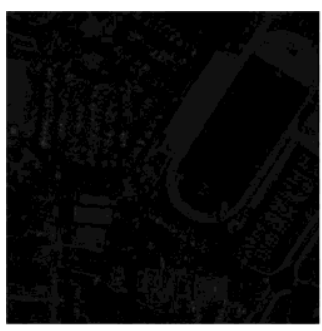

(a)

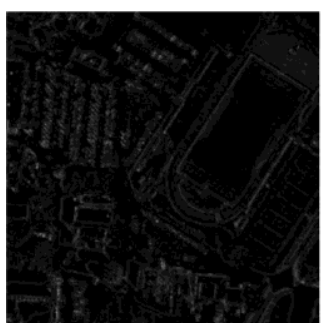

(c)

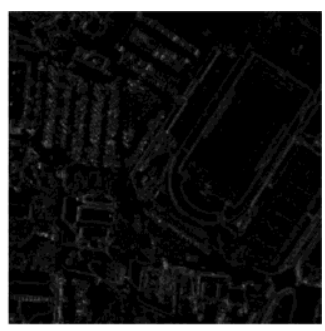

(b)

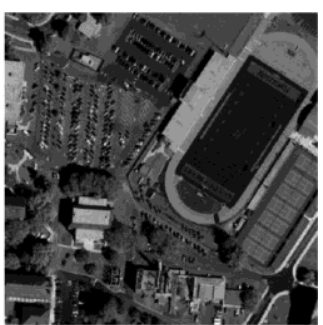

(d)

Fig.: Difference between (d) the original high resolution satellite image and (a)the proposed enhanced image,(b)the standard bicubic interpolation, and (c)the WZP and CS based image resolution enhancement technique. 


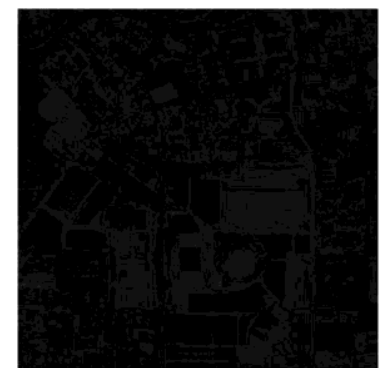

(a)

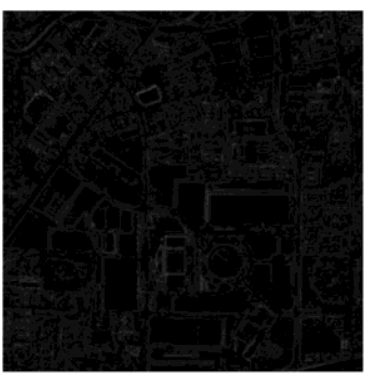

(c)

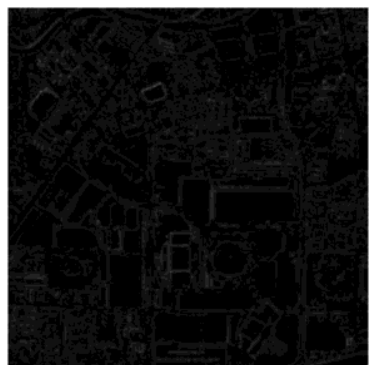

(b)

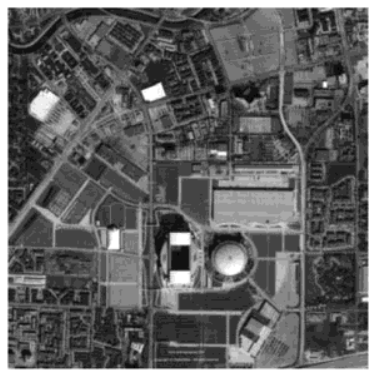

(d)

Fig. Difference between (d)the original high resolution satellite image and (a) the proposed enhanced image,(b) the standard bicubic interpolation, and (c) the WZP and CS based image resolution enhancement technique.

Figs.(a)-(c) show the difference between the high- resolution images with the enhanced image by using the proposed resolution enhancement technique, the difference obtained by using bicubic interpolation directly, and the difference image with WZP- and CS-based image resolution enhancement technique, respectively. Figs and (a) shows that more high-frequency components have been preserved in the proposed technique.

\section{Image interpolation}

Interpolation is a basic tool used extensively in tasks such as zooming, shrinking, rotating, and geometric corrections. Our principal objective in this section is to introduce interpolation and apply image resizing, which are basically image re sampling methods. Uses of interpolation in applications such as rotation and geometric corrections.

Interpolation is the process of using known data to estimate values at unknown locations. We begin the discussion of this topic with a simple example. Suppose that an image of size $500 \times 500$ pixels has to be enlarged 1.5 times to $750 \times 750$ pixels. A simple way to visualize zooming is to create an imaginary $750 \times 750$ grid will be less than the pixel spacing as the original, and then shrink it so that it fits exactly over the original image. Obviously, the pixel spacing in the shrunken 750 X 750 grid will be less than the pixel spacing in the original image. there are three types of interpolation methods. Nearest neighbor interpolation, bilinear, bicubic interpolation. When we are finished assigning intensities to all the points in the overlay grid, we expand it to the original specified size to obtain the zoomed image. The method just discussed is called nearest neighbor interpolation because it assigns to each new location the intensity of its nearest neighbor in the original image. Interpolation is the process of increase the number of pixels in a digital image For nearest neighbor interpolation, the output pixel is assigned the values of pixel that the point falls with in. no other pixels are considered.For bilinear interpolation, the output pixel value is a weighted average of pixels in the nearest 2 by 2 neighborhood. Bilinear interpolation takes longer than nearest neighborhood.

For bicubic interpolation, the output pixel value is a weighted average of pixels in the nearest 4 by 4 neighborhood. Bilinear interpolation takes longer than nearest neighborhood and the Bicubic interpolation takes longer than Bilinear interpolation. Bicubic produces smoother edges than other two methods. bicubic interpolation is an extension of cubic interpolation for interpolating data points on a two dimensional regular grid. The interpolated surface is smoother than corresponding surfaces obtained by bilinear interpolation or nearest-neighbor interpolation. Bicubic interpolation can be accomplished using either Lagrange polynomials, cubic splines, or cubic convolution algorithm.In image processing, bicubic interpolation is often chosen over bilinear interpolation or nearest neighbor in image re sampling, when speed is not an issue. In contrast to bilinear interpolation, which only takes 4 pixels $(2 \times 2)$ into account, bicubic interpolation considers 16 pixels $(4 \mathrm{x} 4)$. Images re sampled with bicubic interpolation are smoother and have fewer interpolation artifacts.

The image of an object in a microscope or telescope can be computed by expressing the object-plane field as a weighted sum over 2D impulse functions, and then expressing the image plane field as the weighted 
sum over the images of these impulse functions. This is known as the superposition principle, valid for linear systems. The images of the individual object-plane impulse functions are called point spread functions, reflecting the fact that a mathematical point of light in the object plane is spread out to form a finite area in the image plane (in some branches of mathematics and physics, these might be referred to as Green's functions or impulse response functions).

When the object is divided into discrete point objects of varying intensity, the image is computed as a sum of the PSF of each point. As the PSF is typically determined entirely by the imaging system (that is, microscope or telescope), the entire image can be described by knowing the optical properties of the system. This process is usually formulated by a convolution equation. In microscope image processing and astronomy, knowing the PSF of the measuring device is very important for restoring the (original) image with deconvolution. Quantitative comparisons confirm the superiority of the proposed method. Peak signal-to-noise ratio (PSNR) and root mean square error (RMSE) have been implemented in order to obtain some quantitative results for comparison. PSNR can be obtained by using the following formula

$$
P S N R=10 \log _{10}\left(\frac{R^{2}}{M S E}\right)
$$

where $\mathrm{R}$ is the maximum fluctuation in the input image ( 255 in here as the images are represented by 8 bit, i.e., 8-bit grayscale representation have been used-radiometric resolution is 8 bit); and MSE is representing the MSE between the given input image $\mathbf{I}_{\mathbf{i n}}$ and the original image $\mathbf{I}_{\text {org }}$ which can be obtained by the following:

$$
M S E=\frac{\sum_{i, j}\left(I_{i n}(i, j)-I_{o r g}(i, j)\right)^{2}}{M x N}
$$

where $\mathrm{M}$ and $\mathrm{N}$ are the size of the images. Clearly, RMSE is the square root of MSE, hence it can be calculated by the following:

$$
R M S E=\sqrt{\frac{\sum_{i, j}\left(I_{i n}(i, j)-I_{o r g}(i, j)\right)^{2}}{M x N}}
$$

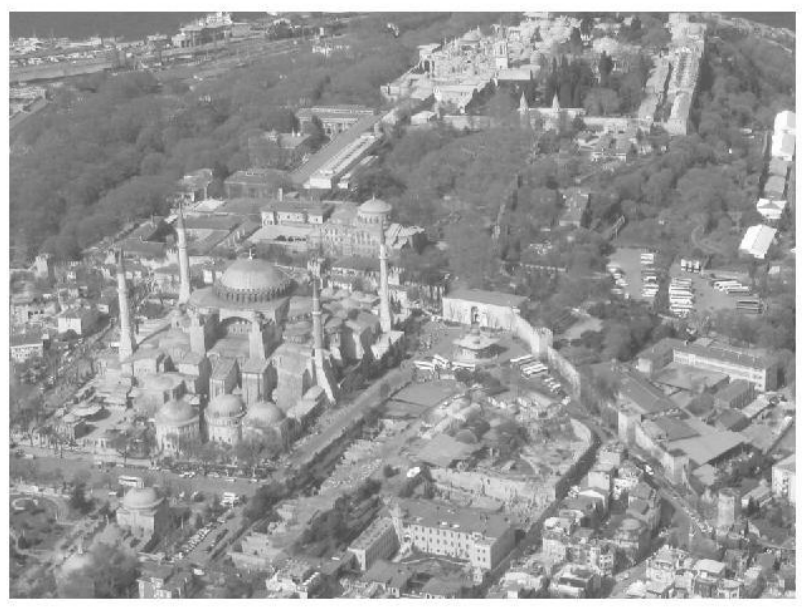

Figure: Low resolution image 


\section{Decomposed Image}
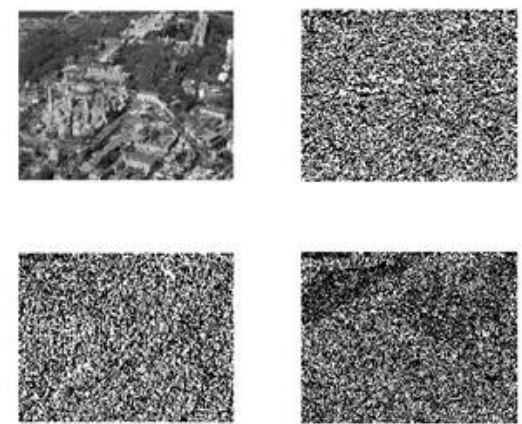

Figure Decomposed image using DWT

\section{Resolution Enhanced Image}

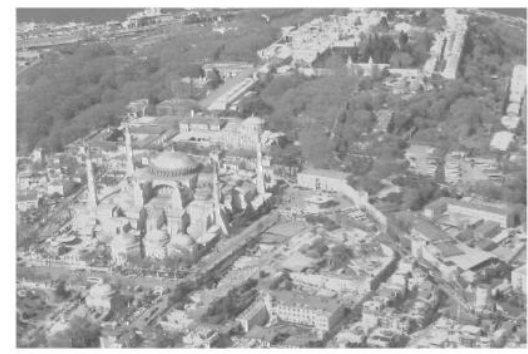

Figure Image enhanced by the proposed technique with enlargement from 702 X 937 to 2808 X 3748

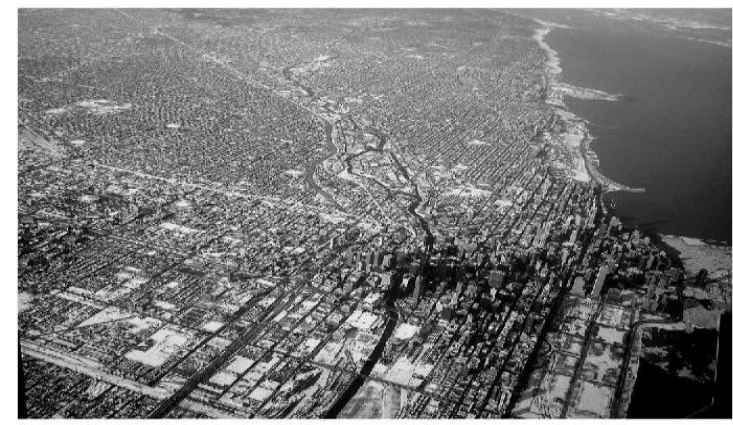

Figure : Low resolution image 

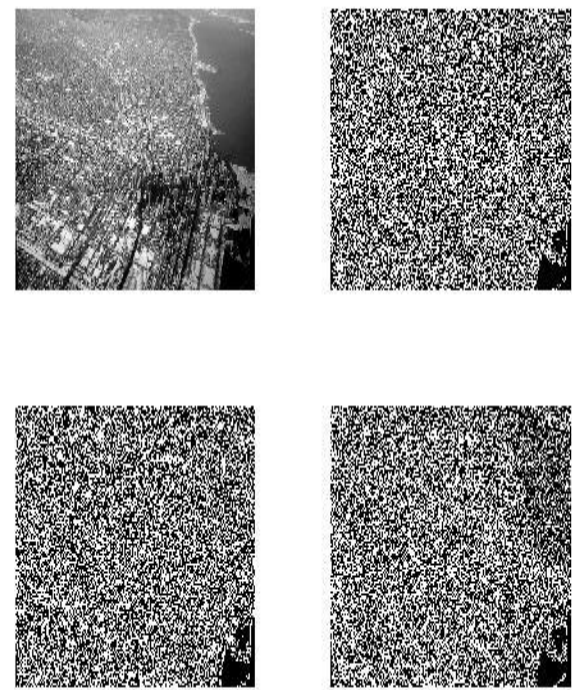

Figure : Decomposed image of fig 5.4 using DWT

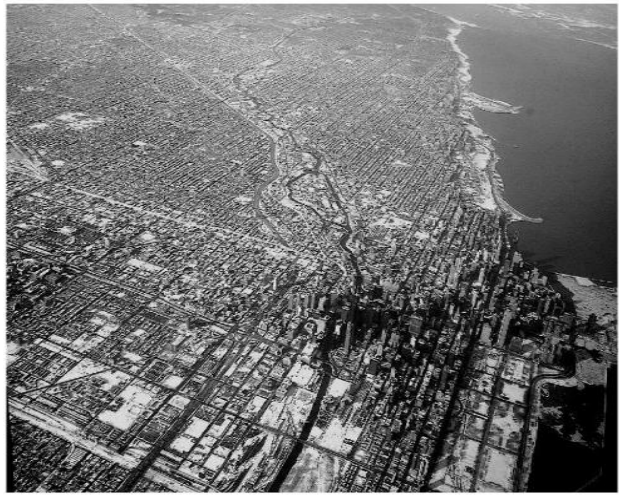

Figure Image enhanced by the proposed technique with enlargement from $768 \times 1024$ to $3072 \times 4096$

\begin{tabular}{|c|c|c|}
\hline $\begin{array}{l}\text { Quantitative } \\
\text { Measures }\end{array}$ & Figure 7.3 & Figure 7.6 \\
\hline PSNR & $32.28 \mathrm{~dB}$ & $39.50 \mathrm{~dB}$ \\
\hline MSE & 38.49 & 7.29 \\
\hline RMSE & 6.20 & 2.70 \\
\hline Entropy & 7.71 & 6.9 \\
\hline
\end{tabular}

Table: Results of fig .1 and 5.4

As expected, highest level of information content is embedded in the original images. The main reason of having the relatively high information content level of the images generated by the proposed method is due to the fact that the unquantized input LL sub band images contain most of the information of the original high-resolution

\section{Conclusion}

This project has proposed a new resolution enhancement technique based on the interpolation of the high-frequency sub band images obtained by DWT and the input image. The proposed technique has been tested on well-known benchmark images, where their PSNR and RMSE and visual results show the superiority of the proposed technique over the conventional and state-of-art image resolution enhancement techniques. The PSNR improvement of the proposed technique is up to $7.19 \mathrm{~dB}$ compared with the standard bicubic interpolation. 


\section{Reference}

[1]. H. Demirel, G. Anbarjafari, and S. Izadpanahi, "Improved motion-based localized super resolution technique using discrete wavelet transform for low resolution video enhancement," in Proc. 17th EUSIPCO, Edinburgh, U.K., Aug. 2009, pp. 1097-1101.

[2]. T. Celik, C. Direkoglu, H. Ozkaramanli, H. Demirel, and M. Uyguroglu, "Region-based super-resolution aided facial feature extraction from low- resolution video sequences," in Proc. IEEE ICASSP, Philadelphia, PA, Mar. 2005, vol. II, pp.789-792.

[3]. H. Demirel and G. Anbarjafari, "Satellite image resolution enhancement using complex wavelet transform," IEEE Geosci. Remote Sens. Lett., vol. 7, no. 1, pp. 123-126, Jan. 2010.

[4]. L. Yi-bo, X. Hong, and Z. Sen-yue, "The wrinkle generation method for facial reconstruction based on extraction of partition wrinkle line features and fractal interpolation," in Proc. 4th ICIG, Aug. 22-24, 2007, pp. 933-937.

[5]. Y. Rener J. Wei, and C.Ken, "Downsample-based multiple description coding and post-processing of decoding," in Proc.27th CCC, Jul. 16-18, 2008, pp. 253-256.

[6]. C. B. Atkins, C. A. Bouman, and J. P. Allebach, "Optimal image scaling using pixel classification," in Proc. ICIP, Oct. 7-10, 2001, vol. 3, pp. 864-867.

[7]. Y. Piao, L. Shin, and H. W. Park, "Image resolution enhancement using inter-subband correlation in wavelet domain," in Proc. IEEE ICIP, 2007, vol. 1, pp. I-445-I-448.

[8]. G. Anbarjafari and H. Demirel, "Image super resolution based on in- terpolation of wavelet domain high frequency subbands and the spa-tial domain input image," ETRI J., vol. 32, no. 3, pp. 390-394, Jun. 2010.

[9]. W. K. Carey, D. B. Chuang, and S. S. Hemami, "Regularity-preserving image interpolation," IEEE Trans. Image Process., vol. 8, no. 9, pp. 1295-1297, Sep. 1999.

[10]. X. Li and M. T. Orchard, "New edge-directed interpolation,” IEEE Trans. Image Process., vol. 10, no. 10, pp. 1521-1527, Oct. 2001.

[11]. K. Kinebuchi, D. D. Muresan, and T. W. Parks, "Image interpolation using wavelet based hidden Markov trees," in Proc. IEEE ICASSP, 2001, vol. 3, pp. 7-11.

[12]. M. S. Crouse, R. D. Nowak, and R. G. Baraniuk, "Wavelet-based statisti- cal signal processing using hidden Markov models," IEEE Trans. Signal Process., vol. 46, no. 4, pp. 886-902, Apr. 1998.

[13]. S. Zhao, H. Han, and S. Peng, "Wavelet domain HMT-based image super resolution," in Proc. IEEE ICIP, Sep. 2003, vol. 2, pp. 933936.

[14]. A. Temizel and T. Vlachos, "Image resolution upscaling in the wavelet domain using directional cycle spinning," J. Electron. Imaging, vol. 14, no. 4, p. 040501, 2005.

[15]. A. Gambardella and M. Migliaccio, "On the superresolution of microwave scanning radiometer measurements," IEEE Geosci. Remote Sens. Lett., vol. 5, no. 4, pp. 796-800, Oct. 2008.

[16]. V. A. Tolpekin and A. Stein, "Quantification of the effects of land-cover- class spectral separability on the accuracy of Markovrandom-field-based superresolution mapping," IEEE Trans. Geosci. Remote Sens., vol. 47, no. 9, pp. 3283-3297, Sep. 2009.

[17]. A. Temizel and T. Vlachos, "Wavelet domain image resolution enhance- ment using cycle-spinning," Electron. Lett., vol. 41, no. 3, pp. 119-121, Feb. 3, 2005.

[18]. L. A. Ray and R. R. Adhami, "Dual tree discrete wavelet transform with application to image fusion," in Proc. 38th Southeastern Symp. Syst. Theory, Mar. 5-7, 2006, pp. 430-433.

[19]. A. Temizel, "Image resolution enhancement using wavelet domain hidden Markov tree and coefficient sign estimation," in Proc. ICIP, 2007, vol. 5, pp. V-381-V-384. 